\title{
DISGRETE GROUPS OF MOTIONS
}

\author{
LEON GREENBERG
}

1. Introduction. This paper deals with the discrete groups of rigid motions of the hyperbolic plane. It is known (12) that the finitely generated, orientation-preserving groups have the following presentations:

Generators:

$$
a_{1}, b_{1}, \ldots, a_{p}, b_{p}, S_{1}, \ldots, S_{d}, c_{1}, \ldots, c_{r} .
$$

Defining relations: $\quad k_{1} \ldots k_{p} S_{1} \ldots S_{d} c_{1} \ldots c_{r}=1$,

$$
S_{1}^{n_{1}}=S_{2}^{n_{2}}=\ldots=S_{d}^{n_{d}}=1
$$

where $k_{m}=a_{m} b_{m} a_{m}^{-1} b_{m}{ }^{-1}$. We shall denote this group by $F\left(p ; n_{1}, \ldots, n_{d} ; r\right)$.

In particular, the finitely generated free groups are contained among these. Indeed, one purpose of this paper is to indicate some geometrical methods for investigating free groups.

The above groups also include the orientation-preserving discrete groups of motions of the sphere and Euclidean plane (3). But the results we shall obtain are mainly concerned with the hyperbolic groups and are either easy or false for the Euclidean and spherical groups. For instance, we shall extend the following theorem of Howson (7) to discrete groups of motions: if $S$ and $T$ are finitely generated subgroups of a free group, then $S \cap T$ is also finitely generated. This theorem is trivial for the Euclidean and spherical groups, which contain no infinitely generated subgroups. We shall generalize the theorem of Karrass and Solitar (8) that if $F$ is a free group and $H$ is a finitely generated subgroup which contains a normal subgroup of $F$, then $H$ is of finite index. This theorem is trivial for the spherical groups and is false for most of the Euclidean groups. For the above reasons we shall consider only the case of discrete hyperbolic groups. We shall usually omit "discrete hyperbolic." We mention the interesting result of Nielsen (11), Bundgaard (1), and Fox (5) that the above groups all contain subgroups of finite index with no elements of finite order.

2. Hyperbolic groups. Let $D$ be the disk $\{z|| z \mid<1\}$ in the complex plane, $\bar{D}$ its closure and $E$ its boundary. $D$ can be given a Riemannian metric so that it becomes the Poincare model of the hyperbolic plane. The geodesics, which we shall call $h$-lines, are arcs of circles orthogonal to $E$. The isometries are the linear fractional transformations which preserve $D$. They are of the forms

Received June 29, 1959. This is in part taken from a doctoral dissertation presented to Yale University. 


$$
S(z)=\frac{a z+\bar{b}}{b z+\bar{a}} \quad \text { and } \quad T(z)=\frac{c \bar{z}+\bar{d}}{d \bar{z}+\bar{c}}
$$

where $a \bar{a}-b \bar{b}=c \bar{c}-d \bar{d}=1$.

The transformation $S$ is called a translation if it has two fixed points which are on $E$. This is equivalent to the condition $|a+\bar{a}|>2$. A translation maps each circle through the fixed points onto itself. In particular, the $h$-line through the fixed points is invariant and is called the axis of the transformation. $S$ is called a rotation if it has a fixed point in $D$, and a limit-rotation if it has a single fixed point on $E$. These conditions are respectively equivalent to $|a+\bar{a}|<2$ and $|a+\bar{a}|=2$. T is a reflection in an $h$-line if $d+\bar{d}=0$. Otherwise $T$ is a glide-reflection, that is, the product of a translation along an axis $\lambda$ with a reflection in $\lambda$.

For each transformation $S$ or $T$, there are a pair of $h$-lines $\lambda$ and $\lambda^{\prime}$, called the isometric circles of the transformation (see (4)). $S$ is the product of a reflection in $\lambda$ and a reflection in the perpendicular bisector of the Euclidean line through the centres of the circles $\lambda$ and $\lambda^{\prime}$. If $T$ is a glide-reflection, this product must be combined with a reflection in the $h$-line through the fixed points of $T$; if $T$ is a reflection, $\lambda=\lambda^{\prime}$ is the $h$-line of reflection. $S$ is a translation, rotation or limit-rotation, according as $\lambda$ and $\lambda^{\prime}$ do not intersect, do intersect, or are tangent. A discrete, hyperbolic group $G$ has a canonical fundamental region, denoted $R_{G}$, which consists of the region in $D$ outside of the isometric circles of all elements of $G$.

A subset $M$ of $\bar{D}$ is called $h$-convex if with every two of its points it contains the $h$-line segment between them. For any subset $M$ of $\bar{D}$, we denote by $[M]$, the $h$-convex closure of $M$, that is, the intersection of all $h$-convex subsets of $\bar{D}$ which contain $M$.

The set of limit points $L_{G}$ of a group $G$ is the intersection with $E$ of the set of limit points of $\{g(z) \mid g \in G\}$, where $z$ is any point in $D$. This set is independent of $z \in D$, because the transformations in $G$ preserve hyperbolic distances, and these become arbitrarily small relative to Euclidean distance, as $E$ is approached. $L_{G}$ is a closed set, invariant under $G$. The convex figure of $G$ is the set $K_{G}=\left[L_{G}\right] \cap D$. This is an $h$-convex set which is invariant under $G$.

For each limit-rotation $g \in G$, it is possible to find a limit-circle $C_{g}$ so that:

(a) $C_{g}$ is tangent to $E$ at the fixed point of $g$,

(b) $C_{g} \subset K_{G}$,

(c) If $g_{1}$ and $g_{2}$ are limit-rotations such that $g_{2}=f g_{1} f^{-1}$, where $f \in G$, then

$$
C_{g_{2}}=f C_{g_{1}},
$$

(d) If $z_{1}$ and $z_{2}$ are two points interior to $C_{g}, u$ is the fixed point of $g$, and $z_{2}=f\left(z_{1}\right)$, where $f \in G$, then $f$ is either a limit-rotation with fixed point $u$, or $f$ is a reflection in an $h$-line with one endpoint at $u$. 
We shall denote by $K^{*}{ }_{G}$ the region obtained from $K_{G}$ by deleting the interior of each $C_{g} . K^{*}{ }_{G}$ is neither unique nor $h$-convex, but it is invariant under $G$. We shall say that $K^{*}{ }_{G}$ is compact $\bmod G$, if there exists a disk $\Gamma=\{z|| z \mid<r$ $<1$ \} such that

$$
K_{G}^{*} \subset G \Gamma=\bigcup_{g \in G} g \Gamma .
$$

This is equivalent to the compactness of the surface obtained from $K^{*}{ }_{G}$ by identifying points congruent under $G$. Nielsen $(\mathbf{1 0} ; \mathbf{1 2})$ has proved that $G$ is finitely generated, if and only if $K^{*}{ }_{G}$ is compact $\bmod G$.

It is not hard to see (by constructing the fundamental region) that every hyperbolic group $F\left(p ; n_{1}, \ldots, n_{d} ; r\right)$ is realized as a group without limitrotations. In fact, according to Nielsen (12), for any finitely generated, hyperbolic group $G$, there is a homeomorphism $s$ of $D$, such that $s G s^{-1}$ is a group of motions without limit-rotations. When $G$ contains no limit-rotations, $K^{*}{ }_{G}=K_{G}$.

3. The results. Coxeter (2) and Goldberg (6) have shown that every abelian subgroup of the modular group $F(0 ; 2,3 ; 1)$ is cyclic. We shall prove the following stronger version of this for the discrete, orientation-preserving, hyperbolic groups.

TheOREM 1. If $F\left(p ; n_{1}, n_{2}, \ldots, n_{d} ; r\right)$ is hyperbolic, then the centralizer of any element is cyclic. The possible finite orders are the divisors of $n_{1}, n_{2}, \ldots, n_{d}$. Any finite subgroup is a cyclic group, conjugate to a subgroup of $\left\langle S_{1}\right\rangle,\left\langle S_{2}\right\rangle, \ldots$, or $\left\langle S_{d}\right\rangle$.

Proof. It is well-known that two orientation-preserving linear fractional transformations commute if and only if they have the same fixed points. Therefore the centralizer of a rotation or limit-rotation is a group of rotations or limit-rotations with the same fixed point, and the centralizer of a translation is a group of translations with the same invariant axis. Each of these groups leaves a curve (or curves) invariant-a circle in $D$, for a group of rotations, a limit-circle for a group of limit-rotations, an $h$-line for a group of translations. Because the group is discrete, there must be an element which transforms a given point (on the invariant curve) the least distance in a fixed direction. This element generates the group, which is therefore cyclic.

The group $F\left(p ; n_{1}, n_{2}, \ldots, n_{d} ; r\right)$ has a fundamental region, which has among its vertices, the points $z_{1}, z_{2}, \ldots, z_{d}$ which are fixed points for $S_{1}, S_{2}, \ldots, S_{d}$ respectively. If $z$ is a fixed point of a rotation $S$, there is an element $f$ which maps $z$ into one of the points $z_{k}$. Then $f S f^{-1}$ is in the subgroup generated by $S_{k}$. Therefore the order of $f S f^{-1}$, which is the same as the order of $S$, divides $n_{k}$.

Let $G$ be any finite subgroup of $F\left(p ; n_{1}, \ldots, n_{d} ; r\right)$, and let $T_{1}$ and $T_{2}$ be two elements (necessarily rotations) with fixed points $t_{1}$ and $t_{2}$ in $D$. We shall show that $t_{1}=t_{2}$. Assuming otherwise, let $\lambda_{3}$ be the $h$-line through $t_{1}$ and $t_{2}$, and $r_{3}$ the reflection in $\lambda_{3}$. There are $h$-lines $\lambda_{2}$ and $\lambda_{1}$ through the points $t_{1}$ 
and $t_{2}$ respectively, such that if $r_{i}$ is the reflection in $\lambda_{i}$, then $T_{1}=r_{2} r_{3}$ and $T_{2}=r_{3} r_{1}$. Therefore $T_{1} T_{2}=r_{2} r_{1}$. If $\lambda_{1}$ and $\lambda_{2}$ diverge, $r_{2} r_{1}$ is a translation; if $\lambda_{1}$ and $\lambda_{2}$ are asymptotic (meet at a point on $E$ ), then $r_{2} r_{1}$ is a limit-rotation. Since these are transformations of infinite order, it follows that $\lambda_{1}$ and $\lambda_{2}$ must meet at a point $t_{3}$ in $D$, and $T_{3}=r_{1} r_{2}$ is a rotation whose fixed point is $t_{3}$. The group $\left\langle r_{1}, r_{2}, r_{3}\right\rangle$ has the triangle $t_{1} t_{2} t_{3}$ as fundamental region. This group is infinite, since the images of $t_{1} t_{2} t_{3}$ under $\left\langle r_{1}, r_{2}, r_{3}\right\rangle$ cover $D$; since $\left\langle T_{1}, T_{2}, T_{3}\right\rangle$ is of index 2 in $\left\langle r_{1}, r_{2}, r_{3}\right\rangle$, the former subgroup is also infinite. We conclude that $t_{1}=t_{2}$, and $G$ is a cyclic group conjugate to a subgroun of $\left\langle S_{1}\right\rangle,\left\langle S_{2}\right\rangle, \ldots$, or $\left\langle S_{d}\right\rangle$.

For hyperbolic groups which contain orientation-reversing transformations, the only exceptions are the following. The centralizer of a translation or glide-reflection can be a product of cyclic groups $C_{\infty} \times C_{2}$. The centralizer of a reflection can be the group $C_{\infty} \times C_{2}$ or $F(0 ; 2,2 ; 1) \times C_{2}$. A finite subgroup can be a dihedral group.

Theorem 2. If $S$ and $T$ are finitely generated subgroups of a discrete group, then $S \cap T$ is also finitely generated.

Proof. Let $H=S \cap T$ and let $G$ be the finitely generated discrete group generated by $S$ and $T$. As we remarked in $\S 2$, we can suppose that $G$ contains no limit-rotations. By Nielsen's theorem, there exist disks

$$
\Gamma_{S}=\left\{z|| z \mid<r_{S}<1\right\}, \quad \Gamma_{T}=\left\{z|| z \mid<r_{t}<1\right\}
$$

such that $K_{S} \subset S \Gamma_{S}$ and $K_{T} \subset T \Gamma_{T}$. Let $r=\max \left(r_{s}, r_{t}\right)$ and $\Gamma=\{z|z|<r\}$. Then

$$
K_{S} \subset S \Gamma \text { and } K_{T} \subset T \Gamma \text {. }
$$

Choose coset representatives $\left\{s_{i}\right\},\left\{t_{j}\right\}$ so that

$$
S=\bigcup_{i} H s_{i} \quad \text { and } \quad T=\bigcup_{j} H t_{j} .
$$

Then

$$
\begin{aligned}
& K_{S} \subset S \Gamma=H \bigcup_{i} s_{i} \Gamma, \\
& K_{T} \subset T \Gamma=H \bigcup_{j} t_{j} \Gamma .
\end{aligned}
$$

Also

$$
K_{H} \subset K_{S} \cap K_{T}
$$

since

$$
L_{H} \subset L_{S} \cap L_{T}
$$

We now show that $s_{i} \Gamma \cap K_{T} \neq \phi$ for only a finite number of representatives $s_{i}$. For any $h \in H, s_{i} \Gamma \cap h t_{j} \Gamma \neq \phi$ if and only if $\Gamma \cap s_{i}{ }^{-1} h t_{j} \Gamma \neq \phi$. Now if $d\left(z_{1}, z_{2}\right)$ is the hyperbolic distance between the points $z_{1}$ and $z_{2}$ in $D$, and the 
hyperbolic radius of $\Gamma$ is $\rho$, then $\Gamma \cap g \Gamma \neq \phi$ if and only if $d(0, g(0))<2 \rho$. But the discreteness of $G$ implies that there are only a finite number of elements $g \in G$ with this last property. Therefore there are only a finite number of elements $g=s_{i}{ }^{-1} h t_{j}$ with $\Gamma \cap g \Gamma \neq \phi$. Note that if

$$
s_{i_{1}}^{-1} h_{1} t_{j_{1}}=s_{i_{2}}^{-1} h_{2} t_{j_{2}} \text {, }
$$

then

$$
s_{i_{2}} s_{i_{1}}^{-1} h_{1}=h_{2} t_{j_{2}} t_{j_{1}}^{-1} \in S \cap T=H .
$$

Therefore $s_{i_{2}} s_{i_{1}}^{-1}$ and $t_{j_{2}} t_{j_{1}}{ }^{-1} \in H$, so

$$
s_{i_{1}}=s_{i_{2}}, \quad t_{j_{1}}=t_{j_{2}}
$$

and $h_{1}=h_{2}$. It follows that there are only a finite number of the $s_{i}, t_{j}, h$ for which $s_{i} \Gamma \cap h t_{j} \Gamma \neq \phi$, and therefore only a finite number of the $s_{i}$ for which $s_{i} \Gamma \cap K_{T} \neq \phi$.

Since $K_{H} \subset K_{T}$, there are only a finite number of the $s_{i}$, say $s_{i_{1}}, s_{i_{2}}, \ldots, s_{i_{n}}$, so that $s_{i} \Gamma \cap K_{H} \neq \phi$. Furthermore, the elements of $H$ map $K_{H}$ and $K_{S}$ onto themselves and consequently $K_{S}-K_{H}$ onto itself. It follows that $s_{i} \Gamma \cap K_{H} \neq \phi$ if and only if $H s_{i} \Gamma \cap K_{H} \neq \phi$. Recalling that

$$
K_{H} \subset H \cup_{i} s_{i} \Gamma
$$

we now obtain

$$
K_{H} \subset H \bigcup_{k=1}^{n} s_{i_{k}} \Gamma
$$

Let $\Gamma^{\prime}$ be a disk with centre 0 and radius $r^{\prime}<1$, which is large enough to contain

$$
\bigcup_{k=1}^{n} s_{i k} \Gamma
$$

Then $K_{H} \subset H \Gamma^{\prime}$, or $K_{H}$ is compact $\bmod H$. Nielsen's theorem now implies that $H$ is finitely generated.

THEorem 3. If $H$ is a finitely generated subgroup of $G$ and if $L_{H}=L_{G}$, then $[G: H]$ is finite.

Proof. If $L_{G}=\phi$, then $G$ must be finite. If $L_{G}$ consists of a single point $z$, then the elements of $G$ and $H$ are limit-rotations whose fixed point is $z$, and possibly reflections in $h$-lines with one endpoint at $z$. It is easy to see that the index $[G: H]$ is finite in this case. If $L_{G}$ contains more than one point, then $K^{*}{ }_{G}$ and $K^{*}{ }_{H}$ are non-empty sets. By Nielsen's theorem there is a disk $\Gamma=\{z|| z \mid<r<1\}$ so that $K^{*}{ }_{H} \subset H \Gamma$. Since $G$ is discrete, there can be only a finite number of elements $g \in G$ so that $\Gamma \cap g \Gamma \neq \phi$. We shall show that every $g \in G$ is congruent $\bmod H$ to one of these elements, which we denote by $g_{1}, g_{2}, \ldots, g_{n}$. Let $z \in \Gamma \cap K^{*}{ }_{H}$ (we suppose that $\Gamma$ is large enough 
so that this intersection is not empty) and let $g \in G$. Since $K_{G}=K_{H}$, we have $K^{*}{ }_{G} \subset K^{*}{ }_{H}$. $K^{*}{ }_{G}$ is invariant under $G$, so $g(z) \in K^{*}{ }_{H}$. Therefore there exists $h \in H$ so that $h g(z) \in \Gamma$. Thus $\Gamma \cap h g \Gamma \neq \phi$ (since $h g(z) \in \Gamma \cap h g \Gamma$ ) and $h g=g_{k}$ for some $k$. It follows that $[G: H]$ is finite.

The following is proved in (4, p. 43).

LEMma 1. If $S$ is a closed subset of $E$ which contains more than one point, and $S$ is invariant under a group $G$, then $S \supset L_{G}$.

Definition. An $N$-chain of a group $G$ is a sequence of subgroups $G_{1}, G_{2}, \ldots, G_{n}$ such that:

(a) $G_{k} \neq\{1\}(k=1,2, \ldots, n)$,

(b) either $G_{k}$ is a normal subgroup of $G_{k+1}$, or $G_{k+1}$ is a normal subgroup of $G_{k}$.

We shall say that two subgroups $H$ and $K$ are $N$-equivalent if there is an $N$-chain $H=G_{1}, G_{2}, \ldots, G_{n}=K$. A subgroup which is $N$-equivalent to $G$ will be called an $N$-subgroup.

We shall call a group quasi-abelian if it leaves invariant an $h$-line or a point in $D$. Such a group is either abelian or has an abelian subgroup of index $2 . G$ is quasi-abelian if and only if $L_{G}$ consists of 0,1 , or 2 points. The following Lemma shows than an $N$-equivalence class consists entirely of quasi-abelian groups if it contains one such group.

Lemma 2. If $G$ and $H$ are $N$-equivalent subgroups of a discrete group and $G$ is not quasi-abelian, then $L_{G}=L_{H}$.

Proof. Let the $N$-chain be $G=G_{1}, G_{2}, \ldots, G_{n}=H$. We proceed to prove by induction that

$$
L_{G_{k}}=L_{G} \quad(k=1,2, \ldots, n) .
$$

Clearly $L_{G_{1}}=L_{G}$. Assume $L_{G_{k}}=L_{G}$. If $G_{k} \subset G_{k+1}$, then $L_{G_{k}} \subset L_{G_{k+1}}$. On the other hand, $L_{G_{k}}$ is invariant under $G_{k+1}$. For let $g \in G_{k+1}$ and $z_{0} \in L_{G_{k}}$. There is a sequence $\left\{h_{j}\right\} \subset G_{k}$ so that for any $z \in D$,

$$
\lim _{j \rightarrow \infty} h_{j}(z)=z_{0}
$$

Now $g h_{j} g^{-1} \in G_{k}$ and

$$
\lim _{j \rightarrow \infty} h_{j} g^{-1}(z)=z_{0}
$$

so that

$$
\lim _{j \rightarrow \infty} g h_{j} g^{-1}(z)=g\left(z_{0}\right) .
$$

Therefore $g\left(z_{0}\right) \in L_{G_{k}}$, and $L_{G_{k}}$ is invariant under $G_{k+1}$. Since $L_{G_{k}}=L_{G}$ and $G$ is not quasi-abelian, $L_{G_{k}}$ contains more than 2 points. By Lemma 1 , 
Thus

$$
L_{G_{k}} \supset L_{G_{k+1}} \text {. }
$$

$$
L_{G}=L_{G_{k}}=L_{G_{k+1}} .
$$

It remains to consider the case where $G_{k+1}$ is a normal subgroup of $G_{k}$. In this case $L_{G_{k+1}} \subset L_{G_{k}}$. Moreover, in the same manner as above we can show that $L_{G_{k+1}}$ is invariant under $G_{k}$.

We assert that $L_{G_{k+1}}$ contains more than one point. If $L_{G_{k+1}}=\phi$, then $G_{k+1}$ is a finite group. $G_{k+1}$ is either a group of rotations (and possibly reflections) with a common fixed point $z \in D$ or a reflection group of order 2 . In the first case the point $z$ must be invariant under all transformations in $G_{k}$. Then $G_{k}$ is also a finite group, so that

$$
L_{G}=L_{G_{k}}=\phi .
$$

But this implies that $G$ is quasi-abelian. In the second case, $G_{k+1}$ consists of the identity and a reflection $r$ in some $h$-line $\lambda$. The elements of $G_{k}$ leave $\lambda$ invariant. $L_{G_{k}}$ is either empty or consists of the endpoints of $\lambda$. This is true also of $L_{G}$, so that $G$ must be quasi-abelian. If $L_{G_{k+1}}$ contains only a single point $z$, then this point is invariant under $G_{k}$. $G_{k}$ is a group of limit-rotations with limit-centre $z$ (and possibly reflections in $h$-lines with one endpoint at $z)$. Then

$$
L_{G}=L_{G_{k}}=\{z\}
$$

and it follows that $G$ is quasi-abelian.

Lemma 1 now implies that $L_{G_{k+1}} \supset L_{G_{k}}$, so that

$$
L_{G}=L_{G_{k}}=L_{G_{k+1}} .
$$

It now follows that $L_{H}=L_{G_{k}}=L_{G}$.

The previous lemma and Theorem 3 imply the following.

Theorem 4. Let $H$ be a finitely generated $N$-subgroup of a non-quasi-abelian group $G$. Then $[G: H]$ is finite.

LEMмA 3. If $U$ and $V$ are subnormal subgroups of a non-quasi-abelian group, then $U \cap V \neq\{1\}$.

Proof. Let $F_{1}, U_{1}$, and $V_{1}$ be the orientation-preserving subgroups of index 2 in $F, U$, and $V$ respectively. In the proof of Lemma 2, we saw that if an $N$-subgroup of $F$ is a reflection group or order 2 , then $F$ is quasi-abelian. Therefore neither $U$ nor $V$ are reflection groups, so $U_{1}$ and $V_{1}$ are non-trivial, subnormal subgroups of $F_{1}$. There exist normal series

$$
\begin{aligned}
& F_{1} \supset F_{2} \supset \ldots \supset F_{n}=U_{1}, \\
& F_{1} \supset F_{2}^{\prime} \supset \ldots \supset F_{n}^{\prime}=V_{1},
\end{aligned}
$$

where some of the $F_{k}$ or some of the $F_{k}{ }^{\prime}$ might coincide. We shall prove inductively that $F_{k} \cap F_{k}{ }^{\prime}$ is a non-trivial non-abelian group. If $F_{2} \cap F_{2}{ }^{\prime}=\{1\}$, 
then each element of $F_{2}$ commutes with each element of $F_{2}{ }^{\prime}$ But two orientation-preserving transformations commute, if and only if they have the same fixed points. This implies that $F_{2}$ (and $F_{2}{ }^{\prime}$ ) is a commutative group. The elements of $F_{2}$ must be rotations with a common fixed point $z_{1} \in D$, limitrotations with a common fixed point $z_{2} \in E$, or translations with a common axis $\lambda$. Since $F_{2}$ is normal in $F_{1}$, the elements of $F_{1}$ must have the same invariant point or $h$-line. Therefore $F_{1}$ is abelian, and $F$ is quasi-abelian. From this contradiction we conclude that $F_{2} \cap F_{2}{ }^{\prime} \neq\{1\}$. Furthermore $F_{2} \cap F_{2}{ }^{\prime}$ is not abelian, since this together with its normality in $F_{1}$ would imply that $F_{1}$ is abelian. Now suppose that $F_{k} \cap F_{k}{ }^{\prime} \neq\{1\}$ and is non-abelian. $F_{k+1} \cap F_{k}{ }^{\prime}$ and $F_{k} \cap F_{k+1}{ }^{\prime}$ are normal subgroups of $F_{k} \cap F_{k}{ }^{\prime}$. By the same argument as before, we conclude that $F_{k+1} \cap F_{k+1}{ }^{\prime}=\left(F_{k+1} \cap F_{k}{ }^{\prime}\right) \cap\left(F_{k} \cap F_{k+1}{ }^{\prime}\right) \neq\{1\}$ and is not abelian. It now follows that $U \cap V \neq\{1\}$.

THEOREM 5. Let $H$ and $K$ be two non-quasi-abelian subgroups of a discrete group. Then $H$ and $K$ are $N$-equivalent, if and only if there is a non-trivial subgroup $J$ which is simultaneously subnormal in $H$ and $K$.

Proof. The "if" part is obvious; we shall prove the "only if" part. There is an $N$-chain $H=G_{1}, G_{2}, \ldots, G_{n}=K$. The series

$$
G_{1} \supset G_{1} \cap G_{2} \supset G_{1} \cap G_{2} \cap G_{3} \supset \ldots \bigcap_{k=1}^{n} G_{k}
$$

is a normal series. We shall prove inductively that

$$
\bigcap_{k=1}^{m} G_{k}
$$

is a non-trivial, subnormal subgroup of $G_{m}$. This is certainly true for $m=1$; assume that this is true for $m=p$. If $G_{p}$ is a normal subgroup of $G_{p+1}$, then

$$
\bigcap_{k=1}^{p+1} G_{k}=\bigcap_{k=1}^{p} G_{k} \not \neq\{1\} .
$$

Since

$$
\bigcap_{k=1}^{p+1} G_{k}
$$

is subnormal in $G_{p}$, which is normal in $G_{p+1}$, it follows that

$$
\bigcap_{k=1}^{p+1} G_{k}
$$

is subnormal in $G_{p+1}$. Now suppose that $G_{p+1}$ is a normal subgroup of $G_{p}$. $G_{p}$ cannot be quasi-abelian. The conditions of Lemma 3 are fulfilled, with

$$
F=G_{p}, \quad U=\bigcap_{k=1}^{p} G_{k}, \quad V=G_{p+1} \cdot
$$

Therefore 


$$
\bigcap_{k=1}^{p+1} G_{k} \neq\{1\}
$$

Since

$$
\bigcap_{k=1}^{p} G_{k}
$$

is subnormal in $G_{p}$,

$$
\bigcap_{k=1}^{p} G_{k} \cap G_{p+1}
$$

is subnormal in $G_{p} \cap G_{p+1}=G_{p+1}$. It now follows that the group

$$
J=\bigcap_{k=1}^{n} G_{k}
$$

is a non-trivial, subnormal subgroup of $H$ and $K$.

This theorem implies that if $G$ is not quasi-abelian, then a subgroup $H$ is an $N$-subgroup if and only if it contains a subnormal subgroup of $G$.

Theorem 6. Let $H$ be a finitely generated non-quasi-abelian subgroup of $G$. Then there is a subgroup $G_{H}$ of $G$ such that

(a) $G_{H}$ is $N$-equivalent to $H$,

(b) if $K \subset G$ and $K$ is $N$-equivalent to $H$, then $K \subset G_{H}$,

(c) $\left[G_{H}: H\right]$ is finite.

Proof. Let $G_{H}=\left\{g \mid g \in G, g L_{H}=L_{H}\right\}$. Since

$$
H \subset G_{H}, L_{H} \subset L_{G_{H}} \text {. }
$$

Lemma 1 implies that $L_{H} \supset L_{G_{H}}$, so that $L_{H}=L_{G_{H}}$. Theorem 3 now implies that $\left[G_{H}: H\right]$ is finite. From this it follows that $H$ has a finite number of conjugate subgroups in $G_{H}$. The intersection of these conjugate subgroups is a normal subgroup $F$ of finite index in $G_{H}$. Since $G_{H}$ is infinite, $F$ is non-trivial. Therefore the sequence, $G_{H}, F, H$, is an $N$-chain, and $G_{H}$ is $N$-equivalent to $H$. If $K$ is $N$-equivalent to $H$, Lemma 2 implies that $K$ leaves $L_{H}$ invariant, so that $K \subset G_{H}$.

THEOREM 7. Let $H$ and $K$ be finitely generated non-quasi-abelian subgroups of a discrete group. Then the following statements are equivalent:

(a) $H$ and $K$ are $N$-equivalent;

(b) there is a group $J$ which is simultaneously normal and of finite index in $H$ and $K$;

(c) $L_{H}=L_{K}$.

Proof. If (a) is true, then $G_{H}=G_{K}$. (These are the groups introduced in Theorem 6.) Since $H$ and $K$ are of finite index in $G_{H}$, this is also true of $H \cap K$. Therefore $H \cap K$ contains a nontrivial subgroup $J$ which is normal and of finite index in $G_{H} . J$ is also normal and of finite index in $H$ and $K$. This shows that (a) implies (b). 
If (b) is true, then $H$ and $K$ are $N$-equivalent. Therefore $L_{H}=L_{K}$. This shows that (b) implies (c).

Now suppose (c) is true, Then $G_{H}=G_{K}$. It follows that $H$ and $K$ are both $N$-equivalent to $G_{H}=G_{K}$, and hence to each other.

It would be interesting to determine whether there are algebraic conditions equivalent to the condition $L_{H}=L_{K}$, when $H$ or $K$ is infinitely generated.

The following is proved in (9, p. 76).

LEMma 4. Let $U$ and $V$ be two groups such that the isometric circles of $U$ are contained in $R_{V}$ and the isometric circles of $V$ are contained in $R_{U}$. Then the group generated by $U$ and $V$ is the free product $U * V$, and $R_{U * V}=R_{U} \cap R_{V}$.

THEOREM 8. Let $H$ be a finitely generated subgroup of a finitely generated non-quasi-abelian group $G$. Then $[G: H]$ is finite if and only if $H$ is contained in no infinitely generated subgroup of $G$.

Proof. If $H$ is of finite index, then any larger group must also be of finite index, and so it is finitely generated.

Now suppose $H$ is of infinite index. We shall find a subgroup of $G$ which contains $H$ and is infinitely generated. We may assume that $G$ contains no limit-rotations. Then $\bar{R}_{H}$, which cannot be contained in $D$, contains intervals on $E$. We first show that one of these intervals contains points of $L_{G}$ in its interior.

If $L_{H}=L_{G}$, then by Theorem $3[G: H]$ is finite. Thus there is a point $z_{0} \in L_{G}-L_{H}$. Let $z \in R_{H}$; there is a sequence $\left\{g_{n}\right\} \subset G$ such that

$$
\lim _{n \rightarrow \infty} g_{n}(z)=z_{0}
$$

Since $R_{H}$ is a fundamental region for $H$, there is $h_{n} \in H$ so that $h_{n} g_{n}(z)=R_{H}$. The sequence $\left\{h_{n} g_{n}(z)\right\}$ has a subsequence which converges to a point $z_{1} \in \bar{R}_{H} \cap E$. The point $z_{1}$ is a limit point of $G$ and belongs to an interval $I_{1}$ of $\bar{R}_{H} \cap E$. $z_{1}$ might possibly be an endpoint of $I_{1}$. Since $G$ is not quasiabelian, $L_{G}$ consists of more than two points, and hence it is a perfect subset of $E$ (see $(4$, p. 68$)$ ). Thus there is a sequence $\left\{x_{n}\right\}$ in $L_{G}$ which converges to $z_{1}$. Suppose this sequence is outside $I_{1} . z_{1}$ is the endpoint of an isometric circle of an element $h \in H$. The transformation $h$ maps $I_{1}$ outside $\bar{R}_{H} \cap E$, and maps a neighbouring interval, containing almost all of the sequence $\left\{x_{n}\right\}$, onto an interval $I$ of $\bar{R}_{H} \cap E$. Therefore $I$ contains points of $L_{G}$ in its interior.

As is shown in (10), the fixed points of the translations of $G$ are dense in $L_{G}$ in the following sense. If $x, x^{\prime} \in L_{G}$ and $J$ and $J^{\prime}$ are intervals of $E$ which contain $x$ and $x^{\prime}$ respectively, then there is a translation $g \in G$, with a fixed point in each interval. A sufficiently high power $g^{n}$ has isometric circles $\lambda$ and $\lambda^{\prime}$ which intersect $E$ inside $J$ and $J^{\prime}$ respectively. Since the interval $I \subset \bar{R}_{H} \cap E$ contains points in $L_{G}$, it contains an infinite sequence of such points, which we denote by $\left\{y_{1}, y_{1}{ }^{\prime}, y_{2}, y_{2}{ }^{\prime}, \ldots,\right\}$. Let $I_{k}, I_{k}{ }^{\prime}$ be mutually disjoint subintervals of $I$ which contain $y_{k}$ and $y_{k}{ }^{\prime}$ respectively. There is a 
translation $g_{k} \in G$ whose isometric circles $\lambda_{k}$ and $\lambda_{k}{ }^{\prime}$ intersect $E$ inside $I_{k}$ and $I_{k}{ }^{\prime}$ respectively. By Lemma 4 , the group generated by $\left\{g_{1}, g_{2}, \ldots,\right\}$ is a free group $F$ of infinite rank, whose fundamental region $R_{F}$ is the region in $D$ outside of all $\lambda_{k}$ and $\lambda_{k}{ }^{\prime}$. Lemma 4 now implies that the group $K$ generated by $H$ and $F$ is the free product $H * F$. Thus $K$ is an infinitely generated group containing $H$.

THEOREM 9. Let $H$ be a finitely generated subgroup of a non-quasi-abelian group $G$. If $H$ has a non-trivial intersection with every non-cyclic subgroup of $G$, then $[G: H]$ is finite.

Proof. We shall show that $L_{H}=L_{G}$. Since $G$ is not quasi-abelian, $L_{G}$ is a perfect subset of $E$. Let $z \in L_{G}$, and let $I$ be an open interval of $E$ which contains $z$. I contains infinitely many points of $L_{G}$. Choose four of them $z_{1}, z_{1}{ }^{\prime}, z_{2}, z_{2}{ }^{\prime}$. Let $I_{1}, I_{1}{ }^{\prime}, I_{2}, I_{2}{ }^{\prime}$ be non-intersecting subintervals of $I$, which contain $z_{1}, z_{1}{ }^{\prime}, z_{2}, z_{2}^{\prime}$ respectively. As in the proof of Theorem 8 , there are translations $g_{1}$ and $g_{2} \in G$, such that the isometric circles $\lambda_{1}$ and $\lambda_{1}{ }^{\prime}$ of $g_{1}$ intersect $E$ inside $I_{1}$ and $I_{1}{ }^{\prime}$ respectively, and the isometric circles $\lambda_{2}$ and $\lambda_{2}{ }^{\prime}$ intersect $E$ inside $I_{2}$ and $I_{2}^{\prime}$ respectively. The group $K$, generated by $g_{1}$ and $g_{2}$, is a free group of rank 2. By hypothesis, the intersection $H \cap K$ is nontrivial. It follows that $H$ has an element whose fixed points are in $I$. Since this is true for any interval $I$ containing $z$, it follows that $z \in L_{H}$ and $L_{H}=L_{G}$. Theorem 3 now implies the required result.

COROllary. Let $H$ and $K$ be finitely generated non-quasi-abelian subgroups of a discrete group. If $H$ has a non-trivial intersection with every non-cyclic subgroup of $K$, then $[K: H \cap K]$ is finite.

Proof. By Theorem 2, $H \cap K$ is finitely generated. The Corollary now follows from Theorem 9. 


\section{REFERENCES}

1. S. Bundgaard and J. Nielsen, On normal subgroups with finite index in F-groups, Mat. Tids. B (1951), 56-58.

2. H. S. M. Coxeter, On subgroups of the modular group, J. de Math. Pures et App. (1958), 317-319.

3. H. S. M. Coxeter and W. O. J. Moser, Generators and relations for discrete groups, Ergeb. der Math. (1957).

4. L. Ford, Automorphic functions (New York, 1951).

5. R. Fox, On Fenchel's conjecture about F-groups, Mat. Tids. B (1952), 61-65.

6. K. Goldberg, Unimodular matrices or order 2 that commute, J. Washington Acad. Sci., 46 (1956), 337-338.

7. A. G. Howson, On the intersection of finitely generated free groups, J. London Math. Soc., 29 (1954), 428-434.

8. A. Karrass and D. Solitar, Note on a theorem of Schreier, Proc. Amer. Math. Soc., 8 (1957), 696-697.

9. W. Magnus, Discrete groups (New York University Notes, 1952).

10. J. Nielsen, Ueber Gruppen linearer Transformationen, Mitteilungen der Math. Ges. in Hamburg Band VIII (1940), 82-104.

11. - Kommutatorgruppen for det frie product af cykliske grupper, Mat. Tids. B (1948), 49-56.

12. - Nogle grundlaeggende begreber vedrørende diskontinuerte grupper af lineaere substitutioner $i$ en kompleks variabel, Den $11^{\text {te }}$ Skandinaviske Matematikerkongress i Trondheim (1949), 61-70.

Brown University 